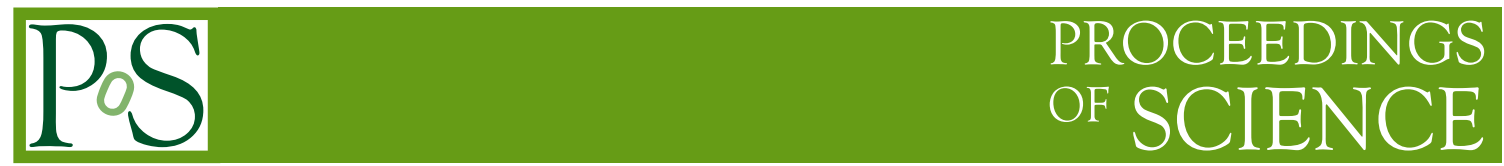

\title{
Measurement of charm fragmentation fractions in photoproduction at HERA
}

\section{Ewald Paul ${ }^{* \dagger}$}

Physikalisches Institut Bonn, Germany

E-mail: paul@physik.uni-bonn. de

The production of charm hadrons in ep scattering at HERA has been measured with the ZEUS detector. Fractions of charm quarks hadronising in particular charm hadron states, called fragmentation fractions, have been determined. They agree with corresponding fractions measured in $e^{+} e^{-}$collisions. This supports the hypothesis that fragmentation fractions are independent of the production process

XXI International Workshop on Deep-Inelastic Scattering and Related Subject -DIS2013, 22-26 April 2013

Marseilles, France

* Speaker.

On behalf of the ZEUS Collaboration 


\section{Introduction}

Fragmentation fractions of charm quarks cannot be predicted by QCD and have to be measured. It is usually assumed that they are universal i.e. independent from the production process. We present a new measurement of charm fragmentation fractions in photoproduction at HERA using a total integrated luminosity of $372 p b^{-1}$. Results are compared to fragmentation fractions obtained with previous HERA data and from $e^{+} e^{-}$storage rings.

\section{Measurement of charm hadrons}

Invariant mass distributions with signals of charm particles are shown in figs. 1, 2, 3, 4 and 5.

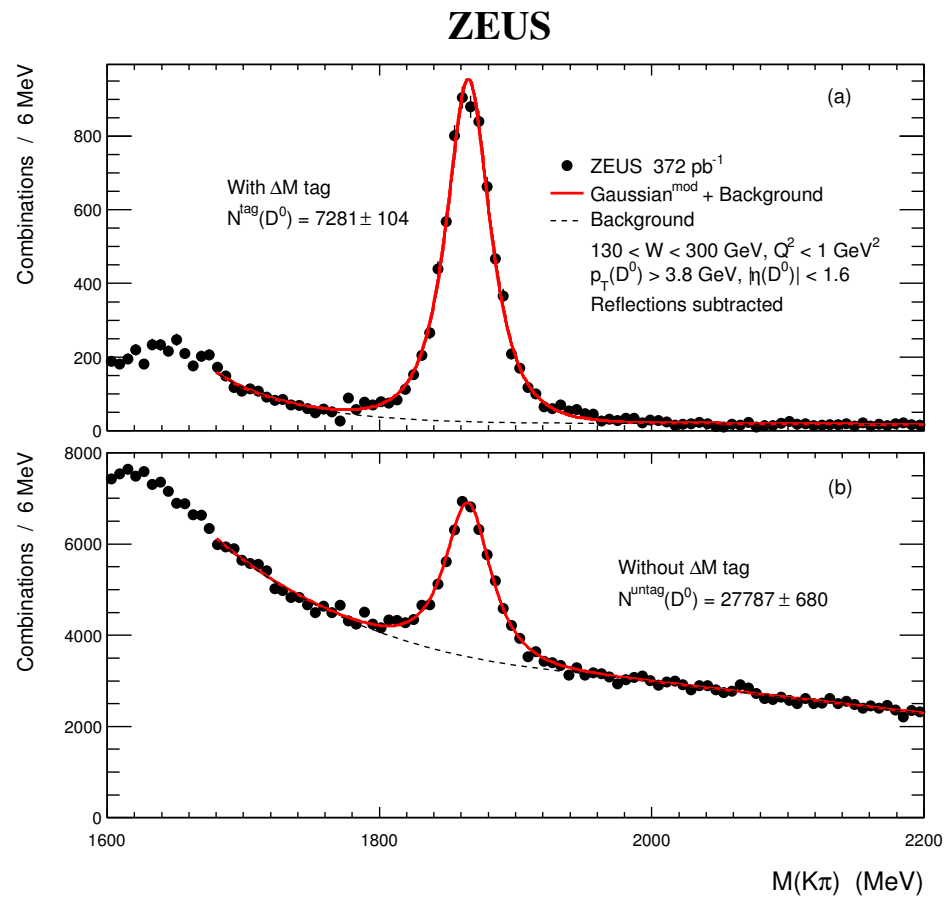

Figure 1: Charm meson $D^{0} / \bar{D}^{0}$ in the $M(K \pi)$ mass distribution, with and without $\Delta M$ tag (see text).

Production yields were measured for the charm hadrons $D^{0} / \bar{D}^{0}, D^{* \pm}, D^{ \pm}, D_{s}^{ \pm}$and $\Lambda_{c}^{ \pm}$in the range of transverse momentum $p_{T}>3.8 \mathrm{GeV}$ and pseudorapidity $|\eta|>1.6$, by fitting a sum of a modified Gaussian for the peaks and empirical parametrisations for the background distributions to the mass distributions. Details of the final data selection and the fits of the charm signals are given elsewhere [1]. The total production rate of $D^{0}$ and $\bar{D}^{0}$ contains two contributions: events "with $\Delta M$ tag" where the mass difference, $\Delta M=M\left(K \pi \pi_{s}\right)-M(K \pi)$, was in the range $0.143<\Delta M<$ $0.148 \mathrm{GeV}$ (fig. 1(a)), and the remaining events"without $\Delta M$ tag" (fig. 1(b)). The $D^{* \pm}$ sample contains the " $\Delta M$ tag" events shown in fig. 1(a) and an additional sample of events from outside the kinematic range in which $D^{0}$ and $\bar{D}^{0}$ were selected (fig. 2). 


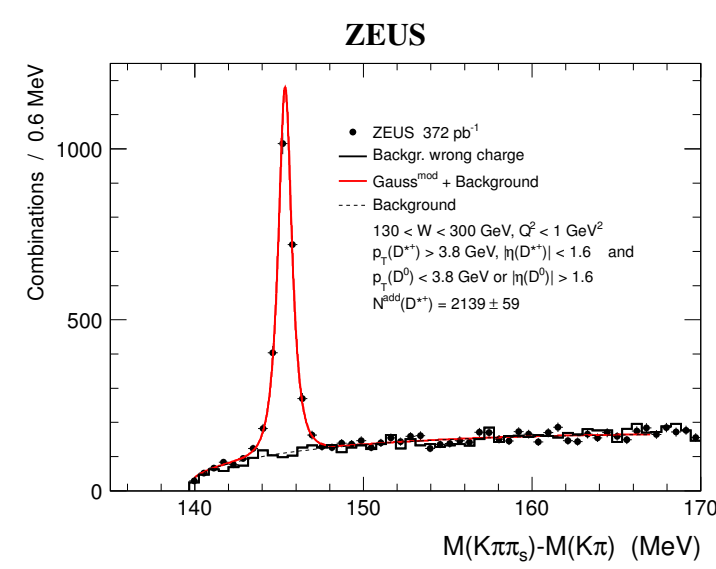

Figure 2: Excited charm meson $D^{* \pm}$ in the difference distribution $M\left(K \pi \pi_{s}\right)-M(K \pi)$.

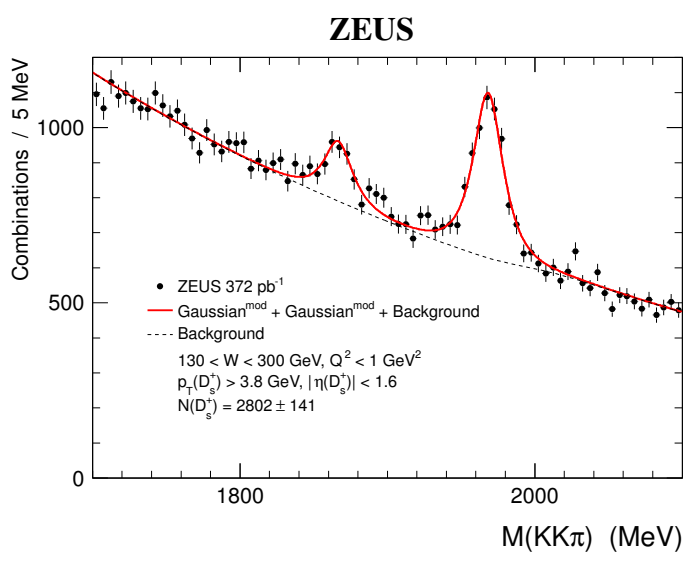

Figure 4: Charm meson $D_{s}^{ \pm}$in the invariant mass distribution $M(K K \pi)$ using the decay mode $D_{s}^{ \pm} \rightarrow \phi \pi^{ \pm}$ with $\phi \rightarrow K^{+} K^{-}$.

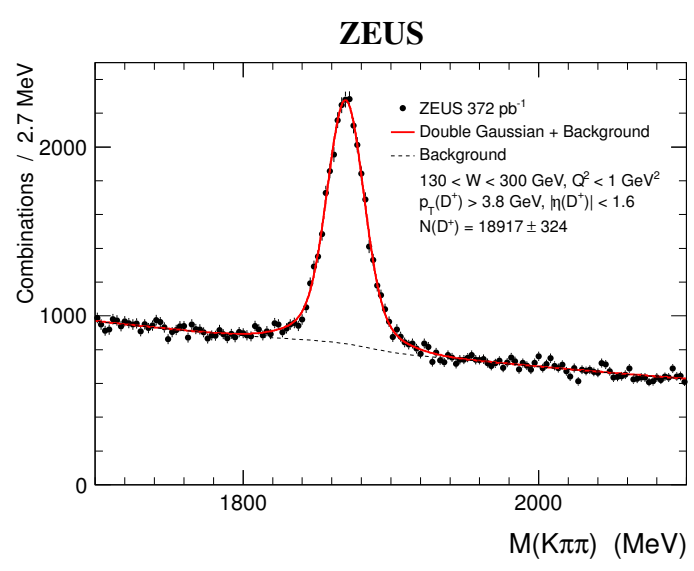

Figure 3: Charm meson $D^{ \pm}$in the invariant mass distribution $M(K \pi \pi)$.

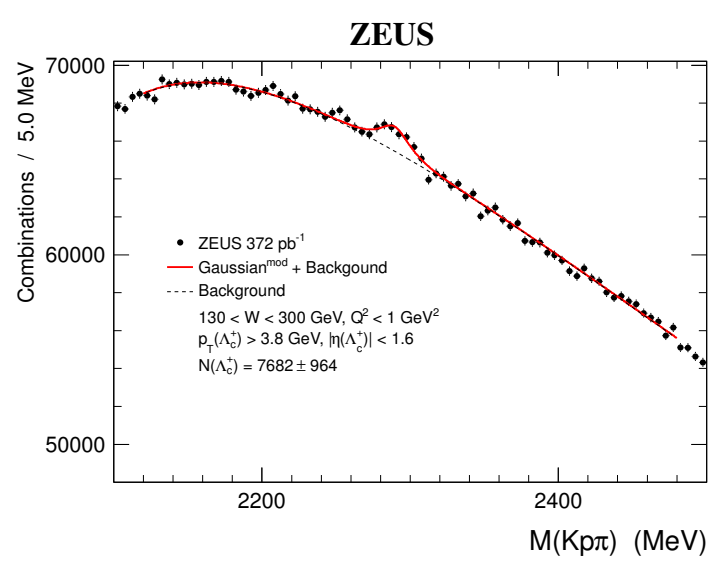

Figure 5: Charm baryon $\Lambda_{c}^{ \pm}$in the invariant mass distribution $M(K p \pi)$.

\section{Systematic uncertainties}

The main systematic uncertainty was caused by the extraction of event rates of charm particles from the fits to the mass distributions. It was estimated by choosing alternative background parametrisations and by variation of the fitted mass range. The total uncertainty varied between below $2 \%$ for $D^{0}$ and $\bar{D}^{0}$ and about $12 \%$ for $\Lambda_{c}^{ \pm}$.

\section{Calculation of cross section ratios}

Charm fragmentation fractions were calculated as ratios of production cross sections for single charm particle production and the sum of all charm particle ground states. Contributions from beauty hadron decays were subtracted using the predictions obtained from PYTHIA. Corrections were applied in order to achieve equivalent phase space treatment: They take into account that 


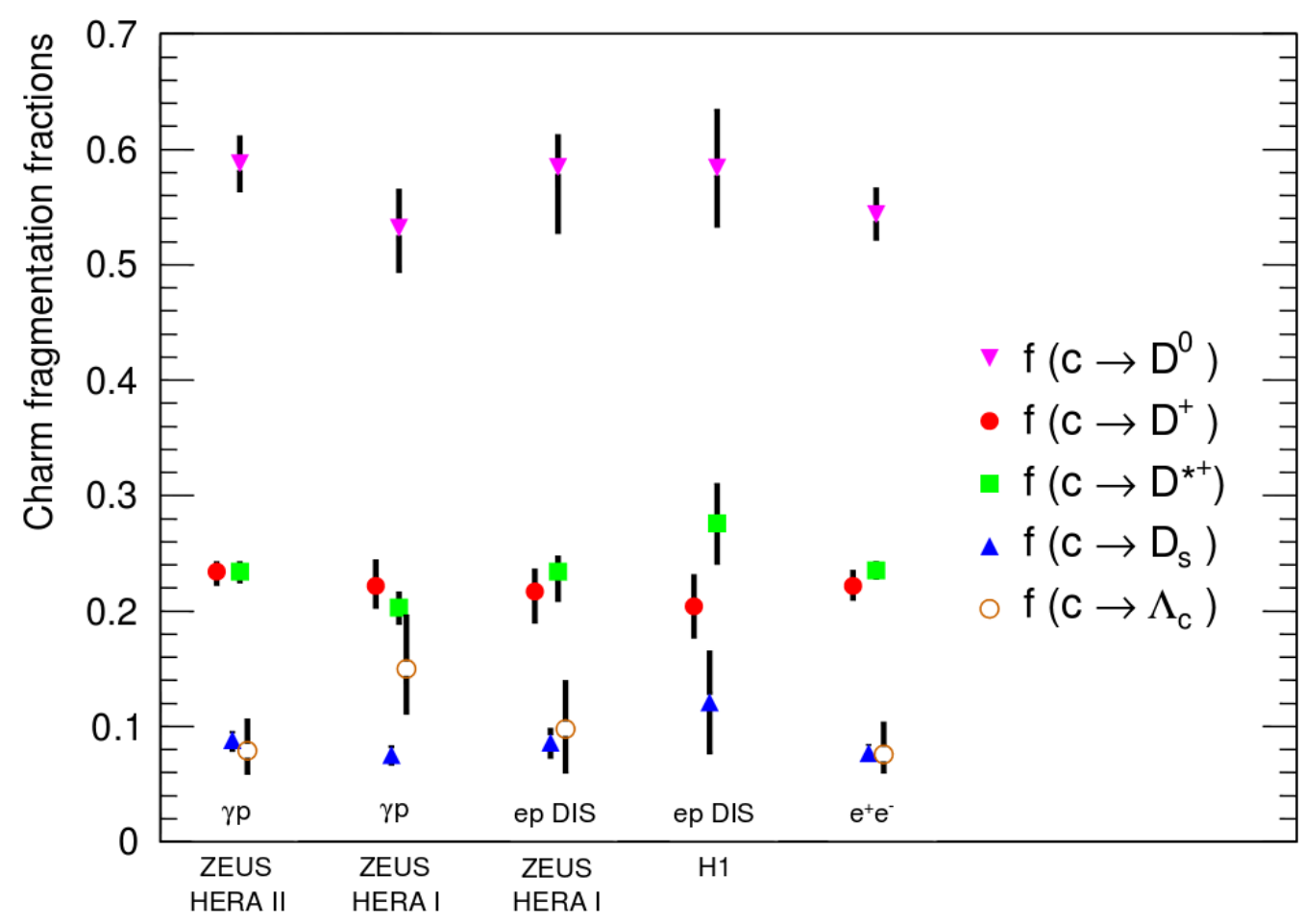

Figure 6: Fractions of charm quarks hadronising in a particular charm hadron . The results of this analysis are shown in the first column. They are compared to other measurments (see text).

only a fraction of the $D^{*}$ momentum was transfered to the daughters $D^{0}, \bar{D}^{0}$ and $D^{ \pm}$, respectively. For the total sum of charm particle cross sections, a correction factor was applied for the not observed states $\Xi_{c}^{ \pm}, \Xi_{c}^{0}$ and $\Omega_{c}^{0}$ and their antiparticles. For the ratios, common uncertainties cancel. Total uncertainties were obtained by adding statisticl, systemtic and branching ratio uncertainties in quadrature.

\section{Fragmentation fractions}

The obtained fragmentation fractions are shown in fig. 6 together with previous results from photoproduction at HERA [2], DIS results at HERA [3, 4] and results from $e^{+} e^{-}$storage rings $[5,6]$. The precision of the fragmentation fractions obtained in this analysis is compatative with that from $e^{+} e^{-}$collssions. The fragmentation fractions of all data sets agree with each other. This demonstrates that fragmentation fractions of charm quarks are independent of the production process and support the hypothesis of universality of heavy quark production.

Two further results have been derived: The rate of charged D' produced in a vector state was $P_{v}^{d}=0.595 \pm 0.020$ (stat. $) \pm 0.025$ (syst. $) \pm 0.011$ (br.), while naive spin counting predicts 0.75 . The strangeness suppression factor defined as $\gamma_{s}=2 \sigma\left(D_{s}^{ \pm}\right) /\left[\sigma^{e q}\left(D^{ \pm}\right)+\sigma^{e q}\left(D^{0} / \bar{D}^{0}\right)\right]$ was determined to be $\gamma_{s}=0.214 \pm 0.013$ (stat. $)_{-0.017}^{+0.006}($ syst. $) \pm 0.012($ br. $)$. This value is fairly consistent with noncharm strange particle production where values between 0.22 and 0.30 were measured. 


\section{Conclusions}

The photoproduction of the charm hadrons $D^{0} / \bar{D}^{0}, D^{* \pm}, D^{ \pm}, D_{s}^{ \pm}$and $\Lambda_{c}^{ \pm}$has been measured with the ZEUS detector. Charm fragmentation fractions were determined. They were found to be consistent with those obtained from $e^{+} e^{-}$collisions and charm productions in DIS at HERA. This supports the hypothesis that heavy-quark fragmentation is universal.

\section{References}

[1] ZEUS Coll, H. Abramovicz et al., Measurement of charm fracmentation fractions in photoproduction at HERA, preprint DESY-13-106 and arXiv: 1306.4862.

[2] ZEUS Coll., S. Chekanov et al., Measurement of charm fracmentation fractions in photoproduction at HERA, Eur. Phys. J. C 44(2005) 351.

[3] H1 Coll., A. Aktas et al., Inclusive photoproduction of $D^{+}, D^{0}, D_{s}^{+}, D^{*+}$ mesons in deep inelastic scattering at HERA, Eur. Phys. J. C 38 (2004) 447.

[4] ZEUS Coll., S. Chekanov et al., Measurement of D mesons Production in Deep Inelastic Scattering at HERA, JHEP 07 (2007) 074.

[5] ZEUS Coll., H. Abramovicz et al., Measurement of $D^{+}$and $\Lambda_{c}^{+}$production in deep inelastic scattering at HERA, JHEP 11 (2010) 1.

[6] L. Gladilin, Experimental results on heavy quark fragmentation., hep-ex/9912064 (1999) .

[7] ATLAS Coll.,ATLAS-CONF. 2011-17 (2011). 